\title{
A Study to Evaluate the Accuracy, Usability, and Acceptance of A Blood-Based HIV Self-Test Performed by Observed Intended Users in Canada
}

Jason Lo Hog Tian

Unity Health Toronto https://orcid.org/0000-0001-9525-8335

Richard A. Galli

Unity Health Toronto

Michelle Sumner-Williams

Unity Health Toronto

Kristin McBain

Unity Health Toronto

\section{Emal Stanizai}

Unity Health Toronto

Wangari Tharao

Women's Health in Women's Hands

Muna Aden

Women's Health in Women's Hands

Heather Jamieson

Hassle Free Clinic

Mark Da Silva

Hassle Free Clinic

Anne-Fanny Vassal

Clinique Medicale L'Actuel

Lorie Guilbault

Clinique Medicale L'Actuel

Laurie Ireland

Nine Circles Community Health Centre

Kim Witges

Nine Circles Community Health Centre

Alexandra King

University of Saskatchewan

Kehinde Ametepee

University of Saskatchewan

Nathan J. Lachowsky 
University of Victoria

\section{Nitika Pant-Pai}

McGill University

\section{Tony Mazzulli}

Public Health Ontario

Sean B. Rourke ( $\sim$ sean.rourke@utoronto.ca )

Unity Health Toronto https://orcid.org/0000-0001-6162-7358

\section{Research article}

Keywords: HIV, blood-based self-test, accuracy, usability, acceptance

Posted Date: October 15th, 2020

DOl: https://doi.org/10.21203/rs.3.rs-89494/v1

License: (c) (i) This work is licensed under a Creative Commons Attribution 4.0 International License. Read Full License

Version of Record: A version of this preprint was published at BMC Public Health on July 18th, 2021. See the published version at https://doi.org/10.1186/s12889-021-11418-z. 


\section{Abstract}

Background: Self-Testing for HIV ("HIVST") is an emerging targeted intervention with the potential to increase the access, uptake and frequency of HIV testing and more effectively reach the undiagnosed especially in priority populations. The study objectives of this study were to (1) evaluate the INSTI HIVSelf-Test (HIV-ST) performance compared with laboratory reference testing, (2) document if intended users can perform the steps to use the HIV-ST device, and (3) document if intended users can successfully interpret contrived positive, negative, and invalid results. Study was intended to be submitted to Health Canada for review for license purposes.

Methods: The study used a cross-sectional design and recruited consenting adults from four community sites across Ontario, Québec, and Manitoba between August 2019 and March 2020. The results of the observed HIV-ST were compared with results of the Abbott Architect HIV Ag/Ab Combo test.

Results: Primary efficacy analysis on 678 completed HIV-ST revealed a positive percent agreement of $100 \%$ and a negative percent agreement of $99.5 \%$ with the comparator method. The overall percent agreement of results interpretation between participant and observer was $93.5 \%$. Of the 708 participants who took part in the usability study, $92.4 \%$ of participants successfully performed the steps determined to be "critical" for successful completion of the test, $96.7 \%$ of participants found the instructions easy to follow, and $95 \%$ of participants indicated that they would use the test again. Of the 404 participants who interpreted the strong positive, weak positive, negative and invalid contrived results, successful interpretation ranged from $90.6 \%$ (for weak positive) to $99.3 \%$ (for negative).

Conclusions: The addition of this very first self-test into the Canadian HIV testing landscape could significantly increase HIV testing rates. An approved blood-based HIV self-test in Canada can offer an accurate, acceptable and simple alternative to facility-based HIV testing, particularly when impacted by Coronavirus pandemic restrictions.

\section{Background}

Unlike other $\mathrm{G} 7$ countries, Canada is not seeing a reduction in the number of new people being diagnosed with HIV. Recent data from the Public Health Agency of Canada (PHAC) indicate that in 2016 an estimated 2,165 people became infected with HIV in Canada, an increase of $10 \%$ over 2014, and it is estimated that there are 63,110 people living with HIV in Canada as of 2016 [1]. Although Canada has endorsed the initial UNAIDS $90-90-90$ target (90\% diagnosed, $90 \%$ of those on treatment and in care, and $90 \%$ of those achieving viral suppression) [2], only $86 \%$ of those living with HIV are diagnosed (1st 90 target) - this represents over 9,000 individuals who have undiagnosed HIV infection across the country who are not adequately served by or connected to our health care system. For those people diagnosed with HIV, 81\% are now on antiretroviral treatment (2nd 90 target), and of those, $91 \%$ have suppressed viral load (3rd 90 target) [1]. While we have reached one of three key UNAIDS targets, there are over 23,000 people who have not yet been diagnosed or who are not on antiretroviral therapy in Canada, which 
represents nearly a third of the total people living with HIV in Canada. Targeted interventions for testing, reaching the undiagnosed, and supporting more people to manage and adhere to treatment, and achieve viral suppression are needed to achieve Canada's UNAIDS commitment to all three of the 90-90-90 targets and move successfully towards ending its HIV epidemic in the next five years. Indeed, diagnosing HIV is critically important for reaching targets aimed at controlling the HIV epidemic [3].

In 2016, WHO recommended HIV Self testing as an alternative to conventional facility-based testing [4]. By 2018, 59 countries had developed national policies on HIV self testing [5].

Self-Testing for HIV ("HIVST") is an emerging targeted intervention with the potential to increase the access, uptake and frequency of HIV testing, and could potentially become a high impact, low cost, and empowering alternative for those who may not otherwise test, notably for populations at high risk for HIV infection [6]. In a review on modern diagnostic technologies for HIV, the authors conclude that these novel technologies, including blood-based HIV self tests, show promise as they are associated with ease of use, high diagnostic accuracy, rapid detection, and the ability to detect HIV-specific markers [7]. There is a growing body of supporting evidence showing the acceptance and usability of HIVST in various global key populations and groups $[6,8,9]$, however similar study data for blood-based HIV self testing within the Canadian population had not existed prior to this study.

Three earlier studies were conducted in Montreal, Quebec with oral HIV self tests in key populations and low risk populations. In these studies, HIV self testing was demonstrated to be an acceptable and feasible testing solution, with data on technology innovations that can help increase testing and expedite linkages to care $[10,11]$. A survey of Canadian stakeholders demonstrated that HIV self testing is desired by Canadians yet, operational models and linkage data need to be thought through [12].

This study aims to provide independent data on the blood-based INSTI HIV Self-Test (HIV-ST) (BioLytical Laboratories, Richmond, BC, Canada) performance, acceptance, and usability in the hands of intended users in Canada The study objectives were to (1) evaluate the device performance, i.e., sensitivity, specificity, and percent agreement compared with laboratory reference testing, (2) document if intended users (non-professional and inexperienced in HIV self-testing) can successfully perform the steps to use the HIV-ST device without product demonstration, and (3) document if intended users can successfully interpret contrived strong positive, weak positive, negative, and invalid results.

\section{Methods}

\section{Study Design}

The study used a cross-sectional design and recruited consenting adults from four community-based sexual health and medical clinics or health centres across Ontario, Québec, and Manitoba between August 2019 and March 2020. Due to the emergence of the COVID-19 infection in populations throughout Canada and related restrictions concerning clinical practice, study sites were forced to discontinue study enrolment by March 18, 2020. The study was open to the English and French-speaking general public 
over the age of 18 who met the inclusion criteria and did not meet any of the exclusion criteria (see Appendix A for full list of inclusion and exclusion criteria). No participant was excluded based on race, gender, ethnicity or sexual orientation. Participants were compensated with $\$ 50$ at their initial study site enrollment and an additional $\$ 25$ when they returned for their laboratory testing results. Self-reported HIV risk activity for the year prior to the study enrolment was collected for each participant. Recruitment occurred during routine clinic visits through use of brochures, waiting room posters, and word of mouth. Sample size for the study was determined through published Health Canada guidelines (Health Canada, 2017).

The results of the HIV-ST performed and interpreted by intended users were compared with results of licensed laboratory-based Comparator Methods (CM). The CM used for all sites was the HIV-1/HIV-2 combination antigen/antibody test (Abbott Architect HIV Ag/Ab Combo test, Abbott Laboratories, Abbott

Park, IL) which is licensed by Health Canada and is in routine use at the central public health laboratories of each of the participating provinces. The Geenius ${ }^{\text {TM }}$ HIV 1/2 Confirmatory Assay (Bio-Rad Laboratories, Marnes-la-Coquette, France) was used for positive confirmation where necessary.

\section{Study Protocol}

After written informed consent was obtained, one venous blood sample was collected from each participant for testing at a central laboratory by the CM. Each participant then started the self-test - they self-collected a fingerstick blood specimen and performed the HIV-ST, including result interpretation, according to only the manufacturer's instructions for use. Each self-test performance was directly observed by a trained healthcare professional (Observer). The Observer did not tutor or interact with the participant conducting the HIV-ST but noted errors and other observations about the participant's test performance. There were 24 items developed, similar to those used in a usability assessment of multiple HIV self-test devices in South Africa [13] to capture usability information including instructions for use, pre-ST preparation, procedure for self-collecting blood sample, self-test procedure, and follow-up procedures. Half of the items (12 in total) were considered "critical" with respect to correct usability. The Observer also interpreted the HIV-ST result immediately after the participant and recorded the result separately. Lastly, a sub-set of participants who volunteered were provided the contrived "mock" result membrane cartridges individually and asked to interpret the results.

The provincial public health laboratories conducted $\mathrm{CM}$ testing on serum from venous blood samples collected from study participants and the results were provided back to the sites and subsequently to the central data coordination centre at St. Michael's Hospital, Toronto. Each central laboratory test result was used for study device performance evaluation with the HIV-ST, however study participants were able to return to the clinic site at a later date to receive their laboratory testing results if desired. All clinical care decisions were based solely on the results of the standard of care HIV testing in place at each site.

\section{Electronic Data Capture}


All participant data were collected by the study observers through the use of pre-loaded tablets at the study sites and stored using Qualtrics (Qualtrics, Provo, UT, USA https://www.qualtrics.com), a survey collection tool that encrypts data using Hypertext Transfer Protocol Secure (HTTPS) and enforces HTTP Strict Transport Security (HSTS). Participant data were stored on local encrypted servers in Toronto at St. Michael's Hospital for further data analysis. Only designated research team members accessed the password-protected data from the Qualtrics servers. The survey data for each participant were coded with a corresponding unique study ID to maintain privacy and confidentiality.

\section{Ethics Approval}

This study was approved by respective Research Ethics Boards (REB) for the Ontario, Québec and Manitoba sites participating in the study: University of Toronto REB, St. Michael's Hospital REB, Veritas IRB Québec, and University of Manitoba HREB. This study was conducted under a Health Canada approved Investigational Testing Authorization (ITA), application No. 276320, issued on December 19, 2018.

\section{Data Analysis}

All data analysis was conducted using IBM SPSS Statistics version 24. The Primary Efficacy Analysis (performance) includes calculation of positive and negative percent agreement between study participants' self-interpreted results of the HIV-ST versus the 4th generation Abbott Architect test results. The proportion of study participants' interpretation of their self-test which is confirmed by the confirmatory test algorithm, and thereby considered "true" will be confirmed following determination of study participant's data inclusion in either the positive percent agreement (PPA) population or the negative percent agreement (NPA) population. Invalid self-test results and results indicated as "do not know" $(n=56)$ were excluded from the calculation of PPA and NPA.

The overall 95\% confidence interval will be determined where:

$P P A=[T P /(T P+F N)] \times 100$, where

TP (true positive) is positive self-test in agreement with positive Architect test, and FN (false negative) is negative self-test discordant with positive Architect test; and

$N P A=[T N /(T N+F P)] \times 100$, where

TN (true negative) is negative self-test in agreement with negative Architect test, and FP (false positive) is positive self-test discordant with negative Architect test.

As a secondary analysis, study participants' self-interpreted results were compared with the self-test results interpreted by the observer.

\section{Results}




\section{Study Participants}

A total of 767 participants were recruited over the study period. Of the 767 participants that started the process, 89 participants were excluded from the Primary Efficacy Analysis according to the exclusion list (see Appendix B for full list of excluded participants). Recruited participant numbers varied across the performance, usability and mock results interpretation arms of the study as not all recruited participants completed all elements.

Table 1 shows the demographic profile of participants included in the primary efficacy analysis $(n=678)$. Over $75 \%$ of the sample was between the ages of 18 and 45 years of age. Over $60 \%$ identified as "male" and $37 \%$ as "female" (for the 21 who chose "other": 8 were "Trans" people, 8 identified as "non-binary", 1 as "agender", 3 as "queer", and 1 as "Two Spirit"). There were two participants who identified that they were pregnant. In terms of ethno-racial composition, approximately $44 \%$ identified as "White", $17 \%$ as "Black", 14\% as "First Nations, Métis or Inuit", 16\% as "Asian", 7\% as "Latin American", and the remaining $2 \%$ as "other". In terms of educational achievement, approximately $27 \%$ of the sample completed High School, $23 \%$ completed College, and $48 \%$ a University degree or higher. Less than $5 \%$ indicated having any kind of reading or writing impairment. In the sample, $89 \%$ identified as "right-handed" and 55\% indicated use of reading glasses. Approximately $45 \%$ were employed, $23 \%$ were unemployed, $13 \%$ were students, $2 \%$ were retired, and $17 \%$ preferred not to answer.

In terms of self-reported HIV status, $20 \%$ indicated that their status was unknown or had never been tested, while $80 \%$ indicated that they were HIV-negative. When asked about HIV testing, $74 \%$ indicated having prior experience with testing. When evaluating self-reported risk category for HIV, 600 participants (89\%) were considered "high risk" for HIV infection. For specific self-reported sexual behaviours in the year prior to survey, $30 \%$ identified as having condomless sex with men while $18 \%$ had condomless sex with women. Approximately $30 \%$ of participants indicated having multiple sexual partners in the past year, 26 individuals (4\%) indicated that their sexual partner was living with HIV, and 51 participants (8\%) indicated having ever injected drugs.

\section{Primary Efficacy (Performance) Analysis}

After removing the 89 subjects who met the exclusion criteria from the 767 recruited participants, the primary efficacy analysis on the 678 who completed HIV-ST revealed a positive percent agreement of $100 \%$ and a negative percent agreement of $99.5 \%$ when comparing the valid self-tester results to the Abbott Architect (see Table 2 for full comparison of participant and laboratory test results and Table 3 for PPA and NPA calculations). The overall percent agreement between participant and observer for the selftest results was $93.5 \%$ (see Table 4 for full comparison of participant and observer interpretations of HIVST results). The overall "invalid" rate for the HIV Self-Test was $5.6 \%$.

\section{Usability Assessment Analysis}


From the total of 767 study participants, 59 subjects withdrew after consenting, did not have a venous blood sample collected or did not complete the self-test, leaving 708 available for analysis with the usability assessment. Overall, the average for all desired outcomes was $91.8 \%$ and for the 12 critical items, the average was $92.4 \%$ (see Table 5 for full breakdown of usability questions). Despite as instructed by the kit instructions for use, only $47.2 \%$ of participants were observed to have washed and dried their hands. Just over $92 \%$ of participants were able to lance their finger correctly, and $88.7 \%$ were able to form a blood droplet, but there was a considerably lower number of participants (81.2\%) who were able to get the blood droplet to fall directly into Bottle 1.

Following the fingerstick blood collection, there were three types of procedures carried out as part of the test process: (1) shaking bottles (accomplished with range of $88.8 \%$ to $90.2 \%$ of time), (2) pouring contents into the test device ( $97.3 \%$ to $99.0 \%$ of participants did so correctly), and (3) waiting for liquids to disappear before adding next liquid into device (participants did this procedure $98.0 \%$ to $98.2 \%$ of the time). Over $89 \%$ of participants performed the procedural steps of the self-test in the correct order, while less than $10 \%$ missed any steps and yet continued the process despite this, and only $1.0 \%$ quit the process before the test was completed.

Table 6 shows the results from the self-test questionnaire given to each participant. Regarding the instructions for use, $96.7 \%$ of participants found them easy to follow. In terms of device use, over $90 \%$ of participants indicated that they were confident with performing the test on their own, and $96.2 \%$ found the device easy to use. Approximately $95 \%$ of participants indicated that they would use the test again and would also recommend this test to a sexual partner or friend. In terms of where participants would prefer using the self-test, $58.6 \%$ indicated that they would do the test at home, while $41.1 \%$ indicated that they would prefer to do the test at a clinic.

\section{Mock Test Interpretation Results}

A total of 404 participants volunteered to complete the mock test interpretation (see Table 7 for a full breakdown of mock test results). Overall, $97.8 \%$ of participants correctly identified a "strong positive", 90.6\% a "weak positive", 99.3\% a "negative" result, 97.8\% an "invalid" (with no control, no test) result, and $93.6 \%$ an "invalid" (with no control, with test) result.

\section{Discussion}

Since data from this study was intended to be submitted by the manufacturer to Health Canada for evidence of the INSTI HIV Self-Test safety and effectiveness as part of the device license application, it was important to determine if the self-test performance met published performance targets. The Health Canada Guidance for Manufacturers of Human Immunodeficiency Virus (HIV) Rapid Diagnostic Tests (RDTs) for use at the Point of Care (POC) or for Self-Testing provides performance targets that must be met for clinical sensitivity and specificity: "Evidence that the RDT intended for use at the POC or for SelfTesting has a minimum sensitivity and specificity of $\geq 99 \%$ for HIV antibody detection should be provided " [14]. Primary efficacy analysis with 678 participants who completed the HIV self-testing study 
revealed a positive percent agreement of $100 \%$ and a negative percent agreement of $99.5 \%$ when comparing the valid self-tester results to the Abbott Architect. The overall specificity, indicated as negative percent agreement of $99.5 \%$, with the lower bound of the $95 \% \mathrm{Cl}$ at $98.6 \%$ meets the required performance criteria.

Of the 6 HIV previously undiagnosed participants living with HIV that were confirmed by the Abbott Architect and Geenius comparator methods, 5 were positive with the self-test and 1 was invalid due to a low volume of fingerstick blood that was used in the INSTI self-test procedure. There was a $93.5 \%$ concordance in the self-test results interpretation between the untrained self-test study participants and the trained observers for the subjects in the primary efficacy analysis. Most discordant results were in the invalid or "do not know/not sure" (uncertainty) response categories.

A total of 38 participants ( $5.6 \%$ of sample) interpreted their self-test results as invalid, including one participant whose venous blood tested positive with the Abbott Architect. Of these, 22 were also interpreted as invalid by the observers, including the one participant living with HIV; however, 15 were interpreted as negative by the observers indicating there was a visible control dot present in the self-test which was either not observed by the self-tester, or not interpreted correctly. In some of these cases, the control dot intensity was indicated to be faint. A faint, but visible control dot may also have led to "do not know/not sure" interpretations recorded by both the self-testers and observers. Mitigations may be needed to improve the fingerstick blood collection process since usability results indicated that nearly 1 in 5 participants had difficulties in getting a free-flowing blood drop to fall into the INSTI sample diluent (Bottle 1). These mitigations could include revisions to the package insert to provide more clear instructions on the proper use of the lancet and subsequent blood drop collection, as well alerting the self-tester that even a faint dot intensity is considered valid. Consequently, self-test users should be instructed to conduct self-testing in a well-lit area.

There was an observed tendency for some self-testers to wipe their fingertip on the rim of the INSTI Bottle 1 (sample diluent) instead of allowing the blood drop to fall freely into the sample diluent. This was indicated for $60 \%(n=22 / 37)$ of invalid self-test results and for several self-test results that were still valid. A further mitigation to address the critical factor for the success in getting the blood drop into Bottle 1 could be to revise the self-test package insert to indicate, with illustration, that one should not scrape or wipe the blood into Bottle 1.

The mock results interpretations for the 404 participants who volunteered to participate in this portion of the study show a high concordance with the expected results. The percent agreement with expected results ranged from $90.6 \%$ for the weak positive result to $99.3 \%$ for the negative result, indicating that untrained self-testers can successfully interpret a range of self-test results including strong positive, weak positive, negative and invalid test results. However, mitigations to the package insert to call attention to the possibility of faint dot intensity could improve the lower success rate in interpreting weak positive results. 
In general, participants expressed a high level of satisfaction with the HIV-ST experience as shown in Table 6: $96.7 \%$ of participants found the instructions for use easy to follow and $96.2 \%$ found the self-test device easy to use. $94.7 \%$ of participants would use the HIV-ST again, and $95.5 \%$ would recommend its use to a sexual partner or friend. Of interest and for consideration - approximately $60 \%$ of participants indicated a preference for using the self-test in a home environment while $40 \%$ would prefer to use the test at a clinic or health care facility, suggesting that both assisted (supervised) and unassisted selftesting strategies will likely be needed for "real world" access and uptake.

Overall, acceptance of the HIV-ST by intended users in this study was high, and similar to studies that reported that oral fluid-based HIV self-testing is highly acceptable among a variety of populations $[6,15$, 16]. Although data on use of blood-based HIV self-tests remains scarce [17-19], performance, usability and acceptance in this study were similar to an INSTI HIV Self-Test study conducted in Kenya [20]. This suggests that populations in both settings were able to conduct and perform the blood-based test as per instructions and agreement and performance metrics were aligned. This attests to the broad use of blood based self-tests as an alternative to oral self-tests.

\section{Conclusions}

The impact of the COVID-19 pandemic and its related public health control measures on HIV treatment and prevention, including access to facility-based testing, has had profound effects on individuals, communities, and societies across the world [21]. Implementation of HIV self-testing has been shown to increase HIV testing rates in men who have sex with men [22], and we found high acceptability amongst the diverse sample of participants in the current study. So, the addition of this HIV self-test into the Canadian HIV testing landscape, once approved by Health Canada, could have similar outcomes. An approved blood-based HIV self-test in Canada can offer an accurate, acceptable and simple alternative to facility-based HIV testing for key populations, particularly when impacted by COVID-19 pandemic restrictions.

\section{List Of Abbreviations}

HIV: Human Immunodeficiency Virus

HIVST: Self-Testing for HIV

HIV-ST: INSTI HIV Self-Test

CM: Comparator Methods

PPA: Positive Percent Agreement

NPA: Negative Percent Agreement 


\section{Declarations}

\section{Ethics Approval and Consent to Participate}

This study was approved by respective Research Ethics Boards (REB) for the Ontario, Québec and Manitoba sites participating in the study: University of Toronto REB, St. Michael's Hospital REB, Veritas IRB Québec, and University of Manitoba HREB. This study was conducted under a Health Canada approved Investigational Testing Authorization (ITA), application No. 276320, issued on December 19, 2018. Informed consent was obtained from all individual participants included in the study.

\section{Consent for Publication}

Not applicable.

\section{Availability of Data and Materials}

The datasets used and/or analyzed during the current study are available from the corresponding author on reasonable request.

\section{Competing Interests}

Richard A. Galli was a paid external contractor for the INSTI HIV Self-Test manufacturer, bioLytical Laboratories, during the early phase of the study conduct.

\section{Funding}

This work was funded by a team grant from the Canadian Institutes of Health Research (FRN 164067). NJL was supported by a Michael Smith Foundation for Health Research Scholar Award (\#16863).

\section{Authors' contributions}

RAG, JL, MSW, KM, ES, WT, MA, HJ, MDS, AFV, LG, LI. KW, KA, and SBR performed the research. RAG, SBR, $\mathrm{JL}, \mathrm{MSW}, \mathrm{KM}$, and ES designed the research study. SBR, RAG, JL, MSW, KM, NJL, TM, and AK analyzed the data. SBR, RAG, and JL wrote the paper.

\section{Acknowledgements}

The authors are grateful to all staff of the Hassle Free Clinic, Toronto; Women's Health in Women's Hands, Toronto; AIDS Committee of Toronto; Clinique Médicale L'Actuel, Montreal; Nine Circles Community Health Centre, Winnipeg; and especially grateful to the staff who participated as study observers: Jane Greer, Leo Mitterni, Nina Malayil, Danayi Munyati, Elmer Bagares, Lisa Ross, Raul Alvarez, Sanbula Zaidi, Setti Tesfu, Zavare Tengra, John Maxwell, Hella Fesehaye, Denese Frans, Natasha Lawrence, Mary Yehdego, Oludolapo Deborah Balogun, Erin Bechamp. 


\section{References}

1. Public Health Agency of Canada. Summary: Estimates of HIV incidence, prevalence and Canada's progress on meeting the 90-90-90 HIV targets, 2016: Government of Canada; 2018 [Available from: https://www.canada.ca/en/public-health/services/publications/diseasesconditions/summaryestimates-hiv-incidence-prevalence-canadas-progress-90-90-90.html

2. Public Health Agency of Canada. Government of Canada announces progress and new investments to eliminate HIV/AIDS as a public health threat: Government of Canada; 2016 [Available from: https://www.canada.ca/en/public-health/news/2016/12/government-canada-announces-progressnew-investments-eliminate-aids-public-health-threat.html.

3. Elliott T, Sanders EJ, Doherty M, Ndung'U T, Cohen M, Patel P, et al. Challenges of HIV diagnosis and management in the context of pre-exposure prophylaxis (PrEP), post-exposure prophylaxis (PEP), test and start and acute HIV infection: a scoping review. Journal of the International AIDS Society. 2019;22(12):e25419.

4. World Health Organization. Policy brief: WHO recommends HIV self-testing 2016 [Available from: https://apps.who.int/iris/bitstream/handle/10665/251549/WHO-HIV-2016.21eng.pdf;jsessionid=D6B8C7E8DA3CB8F2D43C3C6882088629? sequence=1.

5. Unitaid WHO. Market and technology landscape: HIV rapid diagnostic tests for self-testing 2018 [Available from: https://unitaid.org/assets/HIVST-landscape-report.pdf.

6. Johnson C, Baggaley R, Forsythe S, Van Rooyen H, Ford N, Mavedzenge SN, et al. Realizing the potential for HIV self-testing. AIDS and Behavior. 2014;18(4):391-5.

7. Pai N, Karellis A, Kim J, Peter T. Modern diagnostic technologies for HIV. The Lancet HIV. 2020;7(8):e574-e81.

8. Witzel T, Bourne A, Burns F, Rodger A, McCabe L, Gabriel M, et al. HIV self-testing intervention experiences and kit usability: results from a qualitative study among men who have sex with men in the SELPHI (Self-Testing Public Health Intervention) randomized controlled trial in England and Wales. HIV medicine. 2020;21(3):189-97.

9. Kalibala S, Tun W, Cherutich P, Nganga A, Oweya E, Oluoch P. Factors associated with acceptability of HIV self-testing among health care workers in Kenya. AIDS and Behavior. 2014;18(4):405-14.

10. Pai N, Smallwood M, Desjardins L, Goyette A, Birkas KG, Vassal A-F, et al. An unsupervised smart app-optimized HIV self-testing program in Montreal, Canada: cross-sectional study. Journal of medical Internet research. 2018;20(11):e10258.

11. Pai N, Bhargava M, Joseph L, Sharma J, Pillay S, Balram B, et al. Will an unsupervised self-testing strategy be feasible to operationalize in Canada? Results from a pilot study in students of a large canadian university. AIDS research and treatment. 2014;2014.

12. Pai N, Smallwood M, Gulati D, Lapczak N, Musten A, Gaydos C, et al. What do key stakeholders think about HIV self-testing in Canada? Results from a cross-sectional survey. AIDS and Behavior. 2018;22(2):606-15. 
13. Majam M, Mazzola L, Rhagnath N, Lalla-Edward ST, Mahomed R, Venter WDF, et al. Usability assessment of seven HIV self-test devices conducted with lay-users in Johannesburg, South Africa. Plos one. 2020;15(1):e0227198.

14. Health Canada. Guidance Document - Guidance for Manufacturers of Human Immunodeficiency Virus (HIV) Rapid Diagnostic Tests (RDTs) for use at the Point of Care or for Self-Testing: Government of Canada; 2017 [Available from: https://www.canada.ca/content/dam/hcsc/migration/hc-sc/dhp-mps/alt_formats/pdf/md-im/applic-demande/guide-Id/humanimmunodeficiency-virus-rapid-diagnostic-tests-guidance.pdf.

15. Pai N, Sharma J, Shivkumar S, Pillay S, Vadnais C, Joseph L, et al. Supervised and unsupervised selftesting for HIV in high-and low-risk populations: a systematic review. PLoS Med. 2013;10(4):e1001414.

16. Figueroa C, Johnson C, Verster A, Baggaley R. Attitudes and acceptability on HIV self-testing among key populations: a literature review. AIDS and Behavior. 2015;19(11):1949-65.

17. World Health Organization. WHO Prequalification of In Vitro Diagnostics Programme. Product: SURE CHECK HIV self-test 2019 [Available from:

https://www.who.int/diagnostics_laboratory/evaluations/pqlist/191129_pqdx_0054_006_01_sure_check_hiv_self_test.pdf?ua=1.

18. World Health Organization. WHO Prequalification of In Vitro Diagnostics Programme. Product: Mylan HIV self-test 2019 [Available from: https://www.who.int/diagnostics_laboratory/evaluations/pqlist/191003_amended_pqpr_0320_090_00_mylan_hiv_self_test_v2.pdf?ua=1.

19. World Health Organization. WHO Prequalification of In Vitro Diagnostics Programme. Product: INSTI HIV self-test 2019 [Available from: https://www.who.int/diagnostics_laboratory/evaluations/pqlist/191111_amended_final_pqpr_0002_002_01_selftest_v3.pdf?ua=1.

20. Bwana P, Ochieng' L, Mwau M. Performance and usability evaluation of the INSTI HIV self-test in Kenya for qualitative detection of antibodies to HIV. PloS one. 2018;13(9):e0202491.

21. Chenneville T, Gabbidon K, Hanson P, Holyfield C. The Impact of COVID-19 on HIV Treatment and Research: A Call to Action. International Journal of Environmental Research and Public Health. 2020;17(12):4548.

22. Zhang C, Li X, Brecht M-L, Koniak-Griffin D. Can self-testing increase HIV testing among men who have sex with men: a systematic review and meta-analysis. PLoS One. 2017;12(11):e0188890.

\section{Tables}

Table 1 Demographic Profile of Participants included in the Primary Efficacy Analysis $\left(N=678^{*}\right)$ 


\section{Age Range [ $n=678]$}

$18-25(n=139)$ $20.5 \%$

$26-35(n=277)$ $40.9 \%$

$36-45(n=106)$ $15.6 \%$

46-55 ( $n=95)$ $14.0 \%$

$>55(n=61)$

$9.0 \%$

\section{Gender [ $\mathrm{n}=677]$}

Male $(n=410)$

$60.6 \%$

Female $(n=247)$ $36.5 \%$

Other $(n=20)$

$2.9 \%$

\section{Race/Ethnicity [n=678]}

White $(n=296)$ $43.7 \%$

Black $(n=113)$ $16.7 \%$

First Nation, Metis, Inuit ( $n=95)$ $14.0 \%$

South Asian ( $\mathrm{n}=26)$ $3.8 \%$

Southeast Asian $(n=45)$ $6.6 \%$

Arab/West Asian ( $\mathrm{n}=35)$

$5.2 \%$

Latin American $(n=46)$ $6.8 \%$

Other - includes mixed ethnicity $(n=22)$

$3.2 \%$

\section{Highest Education Level [ $n=678]$}

Primary $(n=16)$

$2.4 \%$

Secondary (High School) $(n=184)$

$27.1 \%$

College $(n=156)$

$23.0 \%$

University or Higher $(\mathrm{n}=322)$

$47.5 \%$ 


\section{Reading/Writing Impairment [n=674]}

Yes $(n=30)$

$4.5 \%$

No $(n=644)$

\section{Dominant Hand [ $\mathrm{n}=677]$}

Right $(n=602)$

$88.9 \%$

Left $(n=75)$

$11.1 \%$

Visual Status (Use of reading glasses) [ $\mathrm{n}=678]$

Yes $(n=373)$

$55.0 \%$

No $(n=305)$

$45.0 \%$

\section{Participant is Pregnant [ $n=674]$}

No $(n=672)$

$99.7 \%$

Yes $(n=2)$

$0.3 \%$

\section{Employment Status [n=676]}

Employed $(n=307)$ $45.4 \%$

Unemployed $(n=153)$ $22.6 \%$

Student $(n=87)$ $12.9 \%$

Retired $(n=16)$ $2.4 \%$

Prefer not to answer $(n=113)$

$16.7 \%$

\section{Self-Reported Medical Conditions [ $\mathrm{n}=224]$}

Diabetes $(n=23)$

$10.3 \%$

Hypertension $(n=39)$

$17.4 \%$

Visual impairment $(n=58)$

$25.9 \%$

Existing/Recent sexually transmitted diseases $(n=20)$

$8.9 \%$

Other $(n=84)^{a}$ 


\section{Self-Reported HIV Status [n=677]}

Negative status $(n=540)$

$79.8 \%$

Unknown/Never been tested $(n=137)$

$20.2 \%$

Positive status $(n=0)$

$0.0 \%$

\section{Experience with HIV testing [n=674]}

Yes $(n=500)$

$74.2 \%$

No $(n=174)$

$25.8 \%$

\section{Self-Reported Risk Category $[\mathrm{n}=611]^{\mathrm{b}}$}

Unprotected sex with men $(n=185)$

$30.3 \%$

Unprotected sex with women $(n=108)$

$17.7 \%$

Multiple sexual partners $(n=181)$

$29.6 \%$

Injection drug user $(n=51)$

$8.3 \%$

Born to HIV positive mother $(n=3)$

$0.5 \%$

Sexual partner is HIV positive $(n=26)$

$4.3 \%$

Sexual partner is a bisexual male $(n=46)$

$7.5 \%$

Other $(n=11)^{c}$

$1.8 \%$

\section{Self-Reported Risk [n=678] ${ }^{\mathrm{d}}$}

High risk $(n=600)$

$88.5 \%$

Low/Unknown risk $(\mathrm{n}=178)$

$11.5 \%$

*Category totals below $\mathrm{N}=678$ reflect missing data

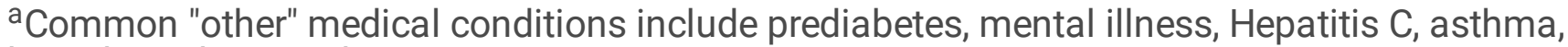
hypothyroidism, and cancer

${ }^{\mathrm{b}}$ Risk categories are mutually exclusive; participants who self-reported multiple risks were put into the highest risk category

cCommon "other" risk categories include "protected sex" and "sex with partner" 
d"High risk" contains participants who self-reported one or more risk category, "low/unknown risk" contains participants who self-reported "other" or no risks

Table 2 Agreement between participant interpreted HIV-ST result and Laboratory Result $(n=678)$

\begin{tabular}{|c|c|c|c|}
\hline \multirow[b]{2}{*}{ Participant Interpreted Result } & \multicolumn{3}{|c|}{ Lab Result } \\
\hline & Negative & Positive & Total \\
\hline Negative & 614 & 0 & 614 \\
\hline Positive & 3 & 5 & 8 \\
\hline Do not know/Not sure & 18 & 0 & 18 \\
\hline Invalid/test did not work & 37 & 1 & 38 \\
\hline Total & 672 & 6 & 678 \\
\hline
\end{tabular}

Table 3 Positive and Negative Percent Agreement with Laboratory Results (Abbott Architect) based on 678 completed HIV Self-Tests

\begin{tabular}{|l|l|}
\hline True Positive & 6 \\
\hline False Negative & 0 \\
\hline True Negative & 669 \\
\hline False Positive & 3 \\
\hline Positive Percent Agreement* & $5 / 5=100 \%(95 \% \mathrm{Cl}: 43.6-97.0 \%)$ \\
\hline Negative Percent Agreement** & $614 / 617=99.5 \%(95 \% \mathrm{Cl}$ : $98.6-99.8 \%)$ \\
\hline $\begin{array}{l}\text { * One additional HIV positive subject (ONO2-015) had an invalid self-test result due to wiping the } \\
\text { fingertip on the rim of bottle } 1 \text { then dropped the bottle and spilled most of the contents before adding } \\
\text { the remainder to the membrane unit. Abbott Architect results were positive. This invalid was not used } \\
\text { in the calculation. }\end{array}$ \\
$\begin{array}{l}* * \text { Participants with invalid self-test results ( } \mathrm{n}=37) \text { and who indicated "do not know" for the self-test } \\
\text { result (n=18) are not included in the calculation }\end{array}$ \\
\hline
\end{tabular}

Table 4 Agreement between self-tester interpreted result and observer interpreted result $(\mathrm{N}=678)$ 


\begin{tabular}{|llllll|}
\hline & \multicolumn{5}{c|}{ Observer Interpreted Result } \\
\hline $\begin{array}{l}\text { Participant } \\
\text { Interpreted Result }\end{array}$ & Negative & Positive & $\begin{array}{l}\text { Do not know / } \\
\text { Invalid/test }\end{array}$ & Total \\
\hline Negative & 602 & 0 & 6 & did not work & \\
\hline Positive & 2 & 5 & 0 & 6 & 614 \\
\hline Do not know/Not sure & 8 & 0 & 4 & 1 & 8 \\
\hline Invalid/test did not work & 15 & 0 & 1 & 6 & 18 \\
\hline Total & 627 & 5 & 11 & 22 & 38 \\
\hline
\end{tabular}

Table 5 Usability Assessment ( $n=708)$ 
Question

Yes

$\%$

\section{Instructions for Use (IFU)}

Did the study participant read/use the Instructions for Use (IFU)?

If yes, was the Instructions for Use (IFU) read before the test?

Was it referred to during the test process?
$99.4 \% \quad 700$

$0.6 \% \quad 4$

$94.6 \% \quad 668$

$5.4 \% \quad 38$

$99.4 \% \quad 695$

\section{Pre-Test}

Did the study participant wash and dry their hands as instructed in the Instructions for Use (IFU)?

$47.2 \% \quad 334 \quad 52.8 \% \quad 374$

Was it difficult for the study participant to remove the test device from the pouch?

Was the study participant able to remove the cap of Bottle 1 ?

Did the study participant twist the tip of the lancet off?

Did the study participant rub his/her finger correctly (up and down/vertical motion)?*

$12.5 \% \quad 88$

$87.5 \% \quad 616$

$99.0 \% \quad 696 \quad 1.0 \% \quad 7$

$99.7 \% \quad 705 \quad 0.3 \% \quad 2$

$88.8 \% \quad 626 \quad 11.2 \% \quad 79$

\section{Blood Draw}

Was the study participant able to lance his/her finger correctly?*

Was the study participant able to form a blood droplet?

$92.1 \% \quad 650$

$7.9 \% \quad 56$

Was the study participant able to get the blood droplet to fall into Bottle 1?*

$88.7 \%$

627

$11.3 \% \quad 80$

Was the study participant able to twist the cap onto Bottle 1?

$81.2 \% \quad 575$

$18.8 \%$

Did the study participant apply bandage?

\begin{tabular}{llll}
$98.7 \%$ & 697 & $1.3 \%$ & 9 \\
\hline $91.4 \%$ & 645 & $8.6 \%$ & 61
\end{tabular}

\section{Self-Test Procedure}

Did the study participant shake Bottle 1, four (4) times?*

$88.9 \% \quad 627$

$11.1 \% \quad 78$

Did the study participant pour all the liquid from Bottle 1 into test device?*

$97.3 \% \quad 686$

$2.7 \% \quad 19$

Did participant wait until liquid from Bottle 1 disappeared before adding liquid from Bottle 2 into device?*

Did the study participant shake Bottle 2, four (4) times?*

$98.0 \% \quad 689$

$2.0 \% \quad 14$

$88.8 \%$

627

$11.2 \%$

79 
$\begin{array}{lllll}\text { Did the study participant pour the liquid from Bottle } 2 \text { into test } \quad 99.0 \% & 699 & 1.0 \% & 7\end{array}$ device?*

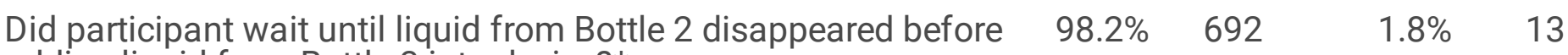
adding liquid from Bottle 3 into device?*

$\begin{array}{lllll}\text { Did the study participant shake Bottle 3, four (4) times?* } & 90.2 \% & 638 & 9.8 \% & 69\end{array}$

$\begin{array}{lllll}\text { Did the study participant pour the liquid from Bottle } 3 \text { into } & 97.3 \% & 687 & 2.7 \% & 19\end{array}$ device and wait until liquid disappeared?*

\section{Procedure Check}

Did the participant quit the process at any point?

$1.0 \% \quad 7$

$99.0 \%$

701

Did the participant perform the steps out of the order?* $10.7 \% \quad 76$

$89.3 \%$

632

Did the participant miss any step and continued the process despite a missed or incorrect step?

$9.5 \% \quad 67$

$90.5 \% \quad 640$

*Critical step

Average on all desired outcomes $=91.8 \%$

Average on critical steps $=92.4 \%$

Table 6 Self-Test Questionnaire $(n=708)$ 


\begin{tabular}{|c|c|c|c|c|}
\hline \multirow[t]{2}{*}{ Question } & \multicolumn{2}{|l|}{ Yes } & \multicolumn{2}{|l|}{ No } \\
\hline & $\%$ & $\mathrm{n}$ & $\%$ & No \\
\hline \multicolumn{5}{|l|}{ Instruction Use } \\
\hline Did you use the test instructions? & $99.4 \%$ & 689 & $0.6 \%$ & 4 \\
\hline Were the instructions for use easy to follow? & $96.7 \%$ & 670 & $3.3 \%$ & 23 \\
\hline Were the pictures and illustrations helpful? & $99.1 \%$ & 685 & $0.9 \%$ & 6 \\
\hline Was the "NOT FOR USERS" section in the IFU helpful? & $44.4 \%$ & 307 & $55.6 \%$ & 385 \\
\hline \multicolumn{5}{|l|}{ Device Use } \\
\hline Was the device easy to use? & $96.2 \%$ & 666 & $3.8 \%$ & 26 \\
\hline Were you confident with performing this test on your own? & $90.6 \%$ & 626 & $9.4 \%$ & 65 \\
\hline \multicolumn{5}{|l|}{ Self-Test Experience } \\
\hline Would you use this test again? & $94.7 \%$ & 655 & $5.3 \%$ & 37 \\
\hline $\begin{array}{l}\text { Would you prefer to use this test at home (yes) or get tested at a } \\
\text { clinic (no)? }\end{array}$ & $58.6 \%$ & 399 & $41.4 \%$ & 282 \\
\hline Would you recommend this test to a sexual partner/friend? & $95.5 \%$ & 658 & $4.5 \%$ & 31 \\
\hline
\end{tabular}

Table 7 Mock Results Interpretation $(n=404)$ 


\begin{tabular}{|c|c|}
\hline Participant Interpretation & $\%$ \\
\hline \multicolumn{2}{|l|}{ Strong Positive } \\
\hline Positive $(n=395)$ & $97.8 \%$ \\
\hline Negative $(n=3)$ & $0.7 \%$ \\
\hline Invalid $(n=5)$ & $1.2 \%$ \\
\hline Do not know $(n=1)$ & $0.2 \%$ \\
\hline \multicolumn{2}{|l|}{ Weak Positive } \\
\hline Positive $(n=366)$ & $90.6 \%$ \\
\hline Negative $(n=7)$ & $1.7 \%$ \\
\hline Invalid $(n=28)$ & $6.9 \%$ \\
\hline Do not know $(n=3)$ & $0.7 \%$ \\
\hline \multicolumn{2}{|l|}{ Negative } \\
\hline Positive $(n=3)$ & $0.7 \%$ \\
\hline Negative $(n=4041$ & $99.3 \%$ \\
\hline Invalid $(n=0)$ & $0.0 \%$ \\
\hline Do not know $(n=0)$ & $0.0 \%$ \\
\hline \multicolumn{2}{|l|}{ Invalid (no control, no test) } \\
\hline Positive $(n=1)$ & $0.2 \%$ \\
\hline Negative $(n=2)$ & $0.5 \%$ \\
\hline Invalid ( $\mathrm{n}=395)$ & $97.8 \%$ \\
\hline Do not know $(n=6)$ & $1.5 \%$ \\
\hline \multicolumn{2}{|c|}{ Invalid (no control, with test) } \\
\hline Positive $(n=9)$ & $2.2 \%$ \\
\hline Negative $(n=11)$ & $2.7 \%$ \\
\hline Invalid $(n=378)$ & $93.6 \%$ \\
\hline
\end{tabular}

Page 22/23 


Do not know $(n=6) \quad 1.5 \%$

\section{Supplementary Files}

This is a list of supplementary files associated with this preprint. Click to download.

- Appendices.docx 